\title{
Double Inversion Recovery Sequence of the Cervical Spinal Cord in Multiple Sclerosis and Related Inflammatory Diseases
}

\author{
I. Riederer, D.C. Karampinos, M. Settles, C. Preibisch, J.S. Bauer, J.F. Kleine, M. Mühlau, and C. Zimmer
}

\begin{abstract}
BACKGROUND AND PURPOSE: MR imaging plays an important role in diagnosing MS and other related inflammatory diseases; however, imaging of the spinal cord is still challenging. We hypothesized that a 3D double inversion recovery sequence for cervical spinal cord imaging would be more sensitive in detecting inflammatory lesions than a conventional 2D T2-weighted TSE sequence at 3T.
\end{abstract}

MATERIALS AND METHODS: On a 3T MR imaging scanner, we examined 30 patients with suspected or established MS (MS, $n=16$; clinically isolated syndrome, $n=12$; isolated myelitis, $n=2$ ) and 10 healthy controls. Newly developed 3D double inversion recovery and conventional 2D axial and sagittal T2-weighted TSE images of the cervical spinal cord were acquired. Two blinded neuroradiologists independently assessed the scans in pseudorandomized order for lesion numbers and rated lesion visibility and overall image quality on 5-point scales. A subsequent consensus reading delivered definite lesion counts. Standardized contrast-to-noise ratios were calculated in representative lesions of each patient.

RESULTS: Overall, 28\% more lesions could be detected with 3D double inversion recovery than with conventional T2WI (119 versus $93, P<$ .002). On average, the standardized contrast-to-noise ratio was significantly higher $(P<.001)$ in double inversion recovery than in $\mathrm{T} 2 \mathrm{WI}$. Lesion visibility was rated significantly higher $(P<.001)$ in double inversion recovery compared with T2WI despite lower image quality.

CONCLUSIONS: The novel 3D double inversion recovery sequence allowed better detection of lesions in MS and related inflammatory diseases of the cervical spinal cord, compared with conventional 2D T2WI.

ABBREVIATIONS: DIR = double inversion recovery sequence; $\mathrm{sCNR}=$ standardized contrast-to-noise ratio

A mong the inflammatory CNS diseases, spinal cord lesions can most frequently be found in multiple sclerosis, though they are very uncommon in other neurologic diseases. ${ }^{1}$ MR imaging of the brain and spinal cord plays a major role not only for establishing the diagnosis of MS but also for evaluating its individual course. $^{2,3}$ The following sequences are recommended in the standard MS spinal cord imaging protocol: 2D (sagittal and axial) proton-attenuation/T2-weighted images, sagittal T1-weighted images pre- and postcontrast, and axial T1-weighted images postcontrast through suspicious lesions. ${ }^{4} \mathrm{MR}$ imaging of the spinal cord, however, is still challenging because of technical difficulties

Received May 13, 2014; accepted after revision July 10.

From the Departments of Diagnostic and Interventional Neuroradiology (I.R., C.P., J.S.B., J.F.K., C.Z.) and Diagnostic and Interventional Radiology (D.C.K., M.S.), Neuroimaging Center at the Technische Universität München (C.P., M.M.), and Department of Neurology (M.M.), Klinikum rechts der Isar, Technische Universität München, Munich, Germany.

Please address correspondence to Isabelle Riederer, MD, Department of Diagnostic and Interventional Neuroradiology, Klinikum rechts der Isar, Technische Univer sität München, Ismaninger Str 22, 81675 Munich, Germany; e-mail:

Isabelle.riederer@tum.de

http://dx.doi.org/10.3174/ajnr.A4093 due to the small size of the spinal cord and its lesions and artifacts caused by deglutition, respiration, and cardiac pulsation. This challenge often leads to uncertainty during assessment of the images. Numerous studies have compared different sequences, such as STIR, which provides high lesion contrast, with this standard protocol in $\mathrm{MS}^{5-7}$ Other studies compared $\mathrm{T}^{*}$ weighted gradient recalled-echo sequences with and without a magnetization transfer prepulse with axial T2 FSE and sagittal STIR images. ${ }^{8}$ In inflammatory spine diseases with a diffuse pattern, such as neuromyelitis optica, diffusion tensor imaging has recently been used to measure fractional anisotropy with great success. ${ }^{9,10}$

The double inversion recovery (DIR) sequence was first described by Redpath and Smith in $1994 .{ }^{11,12}$ In this sequence, the signals from both the CSF and normal white matter are suppressed simultaneously, while inflammatory lesions remain unsuppressed and appear hyperintense. The fast spin-echo version of DIR was introduced 1998 by Bedell and Narayana. ${ }^{13}$ Several studies have shown that this sequence is very sensitive to MS lesions in the brain, especially with respect to intracortical le- 
MRI acquisition parameters of the 3D DIR and axial and sagittal T2WI TSE sequences

\begin{tabular}{lccc}
\hline \multicolumn{1}{c}{ Sequence } & 3D DIR & \multicolumn{2}{c}{ 2D T2WI TSE } \\
\hline Imaging plane & Sagittal & Sagittal & Axial \\
Acquisition matrix & $208 \times 208 \times 300$ & $212 \times 233$ & $308 \times 207$ \\
Acquisition voxel size $\left(\mathrm{mm}^{3}\right)$ & $1.2 \times 1.2 \times 1.3$ & $0.94 \times 1.18 \times 2$ & $0.65 \times 0.88 \times 4$ \\
TR (ms) & 5500 & 3071 & 4238 \\
TE (ms) & 287 & 100 & 100 \\
TSE factor & 173 & 29 & 25 \\
IR delays (ms) & $2550 / 450$ & & \\
Flip/refocusing angle & T2prep with TE $=125 \mathrm{~ms}$ & $90^{\circ} / 120^{\circ}$ & $90^{\circ} / 120^{\circ}$ \\
& and 4 refocusing pulses & & \\
Acquisition time & 7 min 36 sec & 3 min $47 \mathrm{sec}$ & $3 \mathrm{~min} 15 \mathrm{sec}$ \\
Sections & 300 & 15 & 28 \\
\hline
\end{tabular}

Note:-IR indicates inversion recovery; T2prep, preparation pulse to ensure T2 weighting.

sions. ${ }^{14-16}$ An analogous DIR sequence for spinal cord imaging, however, has not yet been established in clinical practice and research. To the best of our knowledge, the only published material on spinal DIR imaging is a vendor demonstration of a DIR sequence of the cervical spinal cord. ${ }^{17}$

The aim of our study was, therefore, to compare a newly established cervical spinal 3D DIR sequence with the conventional axial and sagittal T2WI TSE sequences at $3 \mathrm{~T}$ regarding image quality and lesion detectability. Because the DIR sequence improves the detection of lesions in the brain, especially in the cortex, ${ }^{14-16}$ we hypothesized that the spinal 3D DIR sequence would also be a more sensitive tool for the examination of the spinal cord in inflammatory diseases, especially in patients with suspected or established MS.

\section{MATERIALS AND METHODS}

\section{Patients and Healthy Volunteers}

In 33 patients and 10 healthy volunteers, a DIR sequence was acquired from the cervical spinal cord according to techniques described below. Three of the patients were excluded because of inadequate image quality in 3D DIR (but not in the conventional T2WI TSE) images. Poor image quality in these cases was caused by aliasing artifacts and magnetic field inhomogeneities presumably caused by adiposity (body mass index of $>35$ in all 3 patients). None of the 10 healthy volunteers (4 women; mean age, $33 \pm 4$ years; range, $27-41$ years) had to be excluded. In the remaining 30 patients ( 17 women, mean age, $39 \pm 11$ years; range, 20-57 years), 12 had a prior diagnosis of definite MS, whereas MR imaging was performed in the remaining 18 patients to establish the primary diagnosis. The patients were examined due to a new clinical attack compatible with myelitis or for routine follow-up. Of note, although image data were collected prospectively with the intent of subsequent blinded scientific evaluation, all images were available to clinicians for routine clinical purposes and radiologic reporting immediately after acquisition and, hence, could have impacted clinical diagnoses and decision-making. The final diagnoses were MS $(n=16 ; 11$ relapsing-remitting, 2 primary-progressive, and 3 secondary-progressive), clinically isolated syndrome $(n=12)$, and isolated myelitis of unknown etiology $(n=2)$. The median Expanded Disability Status Scale score was 2.45 (range, 0-7).

Patients were recruited consecutively at the department of neurology at the Klinikum rechts der Isar (Technische Universität München, Munich, Germany), which runs a dedicated center for
MS and related disorders with a high volume of stationary and ambulatory patients. Diagnoses were made by the treating neurologists, experienced experts in MS and inflammatory CNS diseases. The study was conducted in accordance with the 1964 Declaration of Helsinki, and the study protocol was approved by the local ethics committee.

\section{MR Imaging Acquisition}

Scans were performed on a $3 \mathrm{~T}$ scanner (Ingenia; Philips Healthcare, Best, the Netherlands). Our standard protocol consists of multisection sagittal and axial T2-weighted images, sagittal T1-weighted images pre- and postcontrast, and axial T1weighted images postcontrast. In this study, we additionally acquired a 3D fat-saturated double inversion recovery sequence with suppression of the CSF and normal white matter. The acquired voxel sizes were $1.2 \times 1.2 \times 1.3 \mathrm{~mm}$ in the DIR sequence and $0.94 \times 1.18 \times 2 \mathrm{~mm}$ and $0.65 \times 0.88 \times 4 \mathrm{~mm}$ the sagittal and axial T2WI TSE sequences, respectively. The DIR sequence was interpolated to a voxel size of $1.2 \times 1.2 \times 0.65 \mathrm{~mm}$ by zero-filling of the $k$-space. It was then reformatted with section thicknesses of 2 (sagittal) and $4 \mathrm{~mm}$ (axial) for a better comparison with the $2 \mathrm{D}$ sequences. The acquisition times were $7: 36$ minutes (DIR) and 7:02 minutes (T2WI: sagittal, 3:47 minutes; axial, 3:15 minutes). Additional parameters of the sequences are listed in the Table.

\section{MR Imaging Analysis}

Scans were assessed independently by 2 experienced neuroradiologists (I.R. and J.F.K., with 2.5 and 9 years of experience, respectively), blinded to diagnoses and clinical symptoms, on a standard PACS workstation. The 3D DIR and T2WI TSE image series were assessed separately, each in pseudorandomized order, with a temporal delay of at least 2 weeks to exclude biases by memory effects. A lesion was defined as a focal hyperintensity surrounded by a normal isointense spinal cord. Confluent lesions with no distinct delineation in between were counted as 1 lesion. Spinal cord lesions between the top of the $\mathrm{C} 2$ and the bottom of the $\mathrm{C} 7$ vertebral body levels were counted. To assure comparable conditions with the 2D T2WI TSE sequence, we counted lesions in the sagittal and axial reconstructions of the 3D DIR sequence. Additionally, DIR and T2 images were independently rated by the 2 neuroradiologists on 5-point scales in terms of overall image quality, lesion visibility ( 5 , excellent; 4 , good; 3 , average; 2 , poor; 1 , not diagnostic) and artifacts (5, none; 4 , mild, not affecting diagnostic value; 3 , moderate, minor impact expected on diagnostic value; 2, pronounced, major impact on diagnostic value; 1 , severe, no diagnostic value). A definite lesion count was derived from an additional consensus reading held after a delay of at least 1 week by both neuroradiologists, by using the thin-sectioned raw data and coronal reconstructions in addition to the axial and sagittal reconstructions.

The contrast-to-noise ratio comparison between 2 sequences with identical voxel sizes and acquisition durations is traditionally 

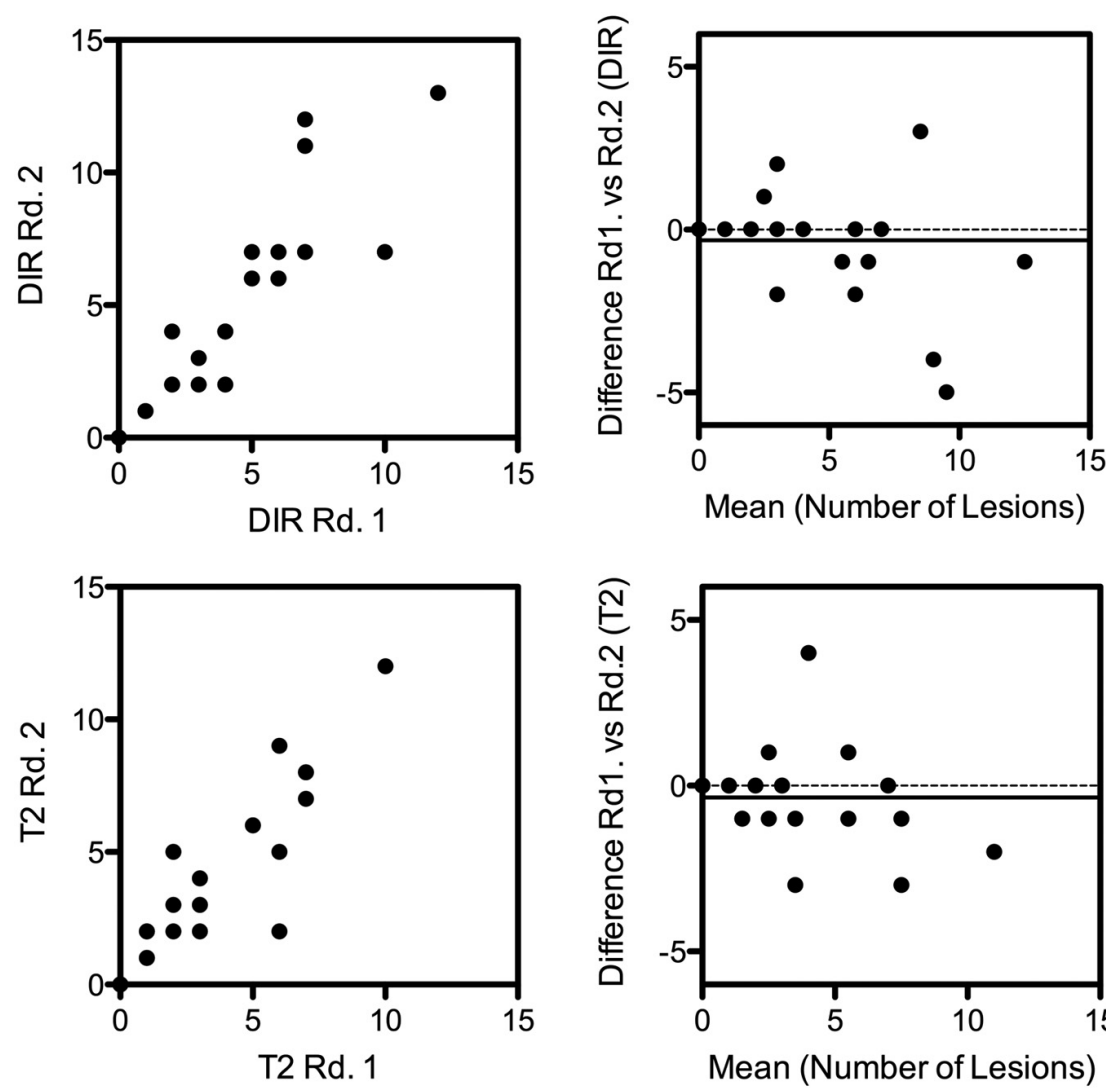

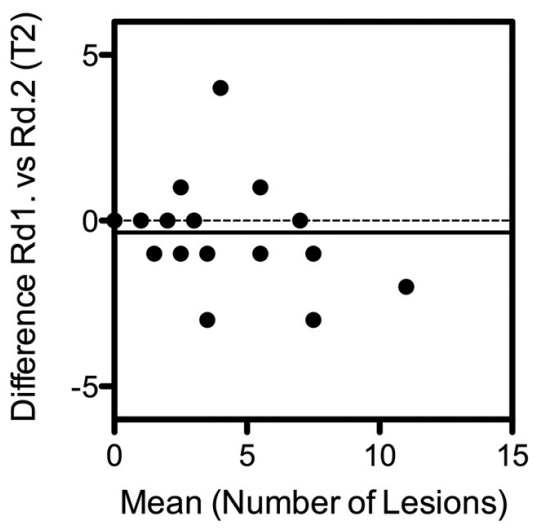

regions with normal signal without any hyperintensities nearby. The noise region was placed in the background in a region without signal and noticeable artifacts.

\section{Statistical Analysis}

Differences in lesion number, sCNR, and image quality were analyzed by using a 2 -sided Student $t$ test for paired samples. The level of significance was set to $P<.01$ for the whole study. The interobserver agreement was calculated by using the Lin concordance (concordance correlation coefficient). ${ }^{19}$ Bland-Altman analysis was performed to assess agreement between the 2 different radiologists for each of the sequences.

\section{RESULTS \\ Lesion Detectability}

In the independent readings, both raters detected substantially more lesions in the reformatted DIR images than in the conventional T2WI TSE images (115 versus $96, P=.097$, and 105 versus $85, P=.004$ ). The slightly higher lesion counts of the more experienced reader were reflected in the Bland-Altman analysis, which showed a similarly small, nonsignificant bias of -0.37 and -0.33 between radiologist 1 and radiologist 2 on T2WI TSE and DIR images, respectively (Fig 1, 95\% CI, $-2.8-2.1$ and $-3.2-2.6$, respectively).

performed on the basis of the ratio of the signal difference between the 2 tissues of interest over the SD of noise. However, as signal noise decreases and contrast-to-noise ratio increases with increasing voxel size and acquisition time, it is questionable to compare (nonstandardized) contrast-to-noise ratios of sequences that are, in these respects, different, as is the case for the 2D T2WI TSE and 3D DIR sequences used. According to Edelstein et al, ${ }^{18}$ the contrast-to-noise ratio is proportional to the acquisition voxel volume and the square root of the total sampling time. To account for this dependency and establish comparability for the 2 sequences, we defined a "standardized" contrast-to-noise ratio (sCNR) according to

$$
s C N R=\frac{S_{\text {lesion }}-S_{\text {spinal cord }}}{S D_{\text {background }} \times V_{\text {voxel }} \times \sqrt{t_{\text {acq }}}},
$$

where $S_{\text {lesion }}$ and $S_{\text {spinal cord }}$ denote the mean signal in a region of interest in the lesion and normal spinal cord tissue, respectively. $S D_{\text {background }}$ is the SD of noise in a region of interest selected in the background, $V_{\text {voxel }}$ is the voxel size, and $t_{a c q}$, the acquisition time in seconds. Calculation and measurement of the mean values were performed with standard tools of the PACS workstation. To calculate the average signal intensity of lesions, we placed circular ROIs in the largest lesion of each patient that was easily discernible in both the T2WI TSE and DIR images. The ROIs for the mean signal intensities of healthy spinal cord tissue were placed in
The concordance between radiologists was similar for both the DIR and T2WI TSE sequence (both, concordance correlation coefficient $=0.91$ ).

In the consensus reading, differences in lesion counts between the DIR and T2WI TSE sequences occurred in 13 patients, always favoring the DIR images. All lesions apparent in the T2WI TSE images were also detected in the DIR images, but not vice versa (Fig 2). Overall, 28\% more lesions could be detected with the 3D DIR sequence than with the conventional 2D T2WI TSE sequence (119 versus 93, $P<.002$ ). On average, 4 lesions per patient were detectable in the DIR sequence, compared with 3 in the conventional T2WI TSE sequence (range, 0-13 [DIR], 0-11 [T2WI TSE]; mean, $3.97 \pm 3.85$ [DIR], $3.10 \pm 3.00$ [T2WI TSE]; $P<.01$; Fig 3).

\section{Image Quality}

The sCNR was significantly higher in the 3D DIR sequence compared with the standard 2D T2WI TSE sequence $(0.36 \pm 0.14$ versus $0.26 \pm 0.14, P<.001)$; thus, the visibility of the lesions was clearly improved in the 3D DIR sequence (Fig 4). On a 5-pointscale, lesion visibility was rated significantly higher in the DIR sequence compared with the T2WI TSE sequence (4.8 versus 3.3 on a 5 -point scale, $P<.001$ ), despite lower overall image quality (3.7 versus $4.8, P<.001$ ).

The DIR sequence seems to be more prone to motion artifacts 

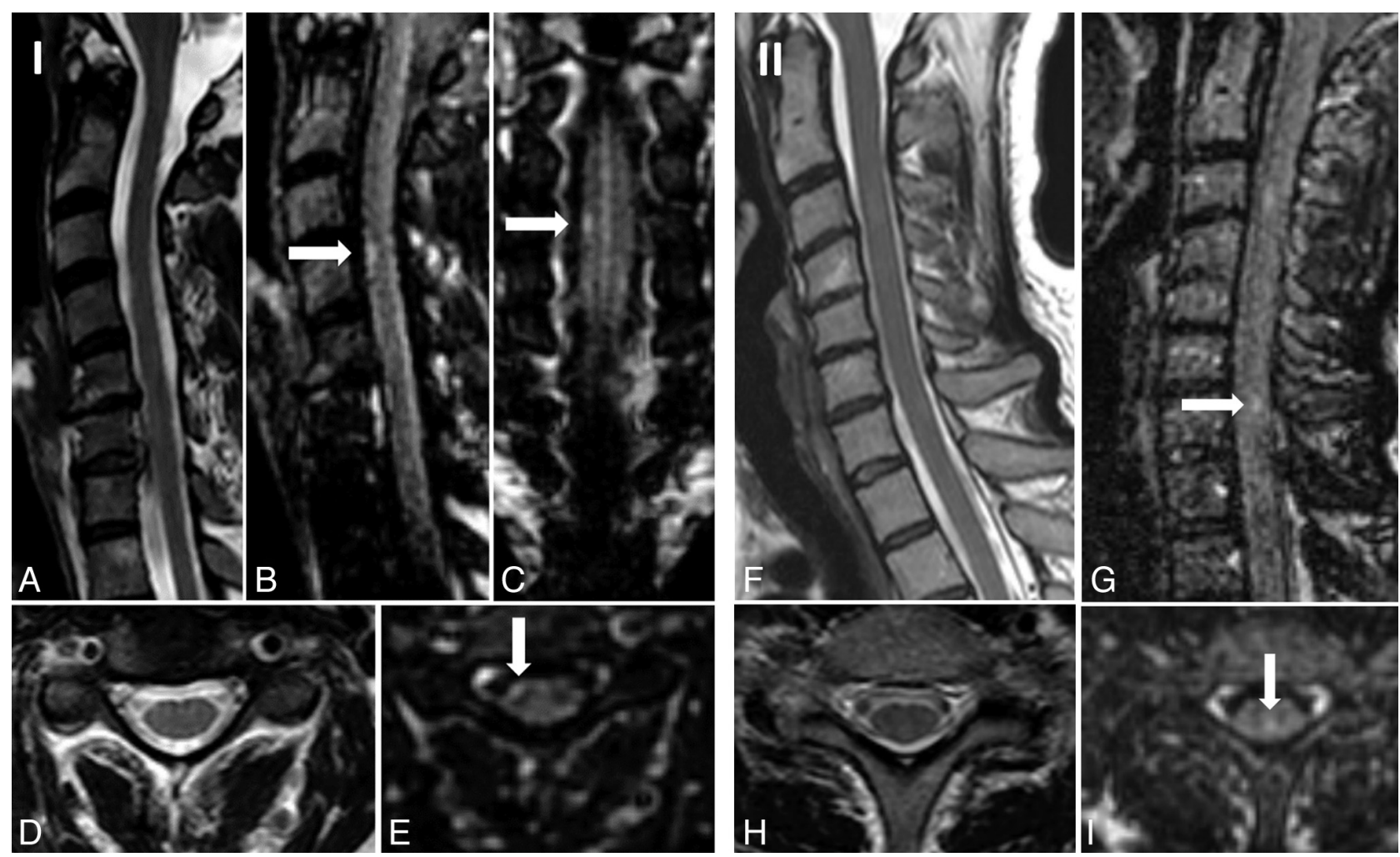

FIG 2. Examples of 2 patients with lesions visible only in the DIR images and not in the T2WI TSE images. Sagittal $(A$ and $F)$ and axial $(D$ and $H)$ T2WI TSE images; sagittal ( $B$ and $G$ ), coronal $(C)$, and axial ( $E$ and $I)$ reconstructions of the 3D DIR sequence of the spinal cord. The group of images on the left (I, A-E) shows the cervical spinal cord of a 52-year-old female patient. Note the lesion in the spinal cord at the C4 vertebral body level, which is only visible in the DIR sequence. This patient indicated pain in her left shoulder, weakness of her left arm, and tingling in her left palm. In T2WI TSE images, 1 lesion was visible in the cervical spinal cord at the C2/C3 level with discrete contrast enhancement (not shown in the image). At first, the differential diagnosis included neoplasm and inflammation. Due to the cervical 3D DIR sequence, another small lesion was detected at the C4 lateral level on the right (arrow), favoring the diagnosis of cervical myelitis. The group of images on the right (II, $F-I)$ shows the spinal cord of a 49-year-old patient with clinically isolated syndrome. Note the lesion in the spinal cord at the C7 vertebral body level, which is only visible in the DIR sequence.

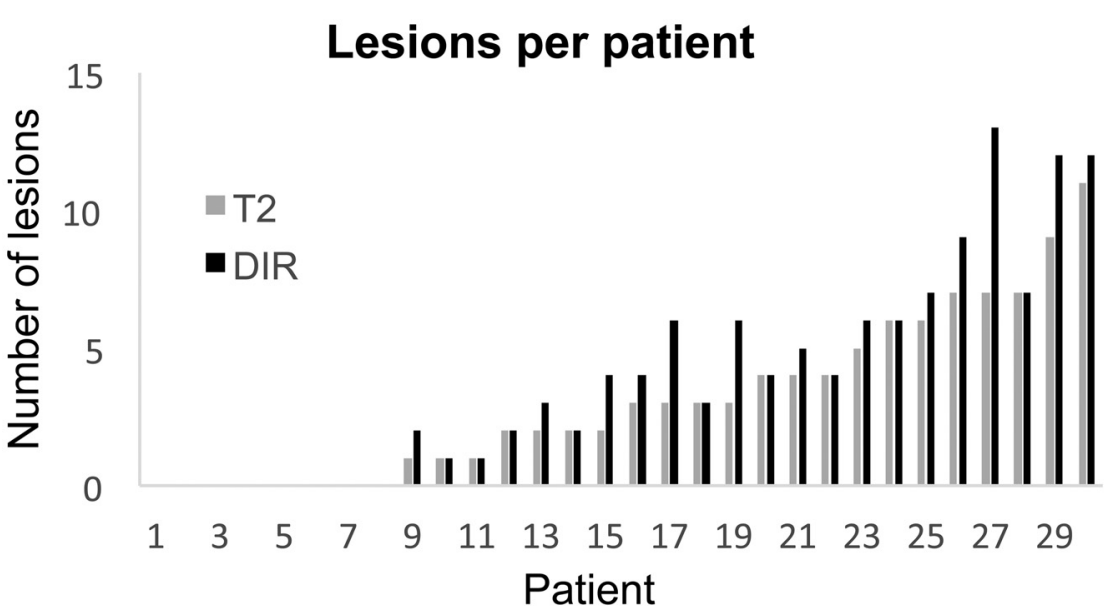

FIG 3. The number of lesions per patient according to the consensus reading of both radiologists in the T2WI TSE and DIR sequences. Volunteers are not included. Range, 0-13 (DIR), 0-11 (T2WI TSE); mean, $3.97 \pm 3.85$ (DIR), $3.10 \pm 3.00$ (T2WI TSE); $P<.01$.

such as swallowing. Eight T2WI TSE images but only 2 DIR images were rated as completely free of artifacts (4.5 versus 3.8, $P<$ $.001)$. Furthermore, image quality of the DIR sequence was restricted in 3 patients due to aliasing artifacts and B1 inhomogeneities due to obesity. Therefore, these patients were excluded from the study. No false-positive lesions of the spinal cord were found in the control group in any of the sequences.

\section{DISCUSSION}

The present study demonstrates that the DIR sequence, which has been shown to improve visibility of MS-related inflammatory lesions, in particular of intracortical lesions in the brain, ${ }^{14,15}$ can be adapted for imaging of the cervical spinal cord. The newly developed spinal 3D DIR sequence is more sensitive than conventional T2WI TSE sequences for inflammatory lesions in patients with suspected or definite MS. This sensitivity can be attributed to the higher sCNR of the 3D DIR sequence and the correspondingly enhanced lesion visibility.

Early attempts to improve spinal cord imaging in MS strived to establish a spinal version of the fluid-attenuated inversion recovery sequence, trying to take advantage of the high sensitivity of the FLAIR sequence for MS lesions in the brain. ${ }^{6,20,21}$ However, due to poor contrast between lesions and healthy spinal cord, the sensitivity of the spinal FLAIR sequence was actually lower compared with conventional T2-weighted images. ${ }^{20}$ 

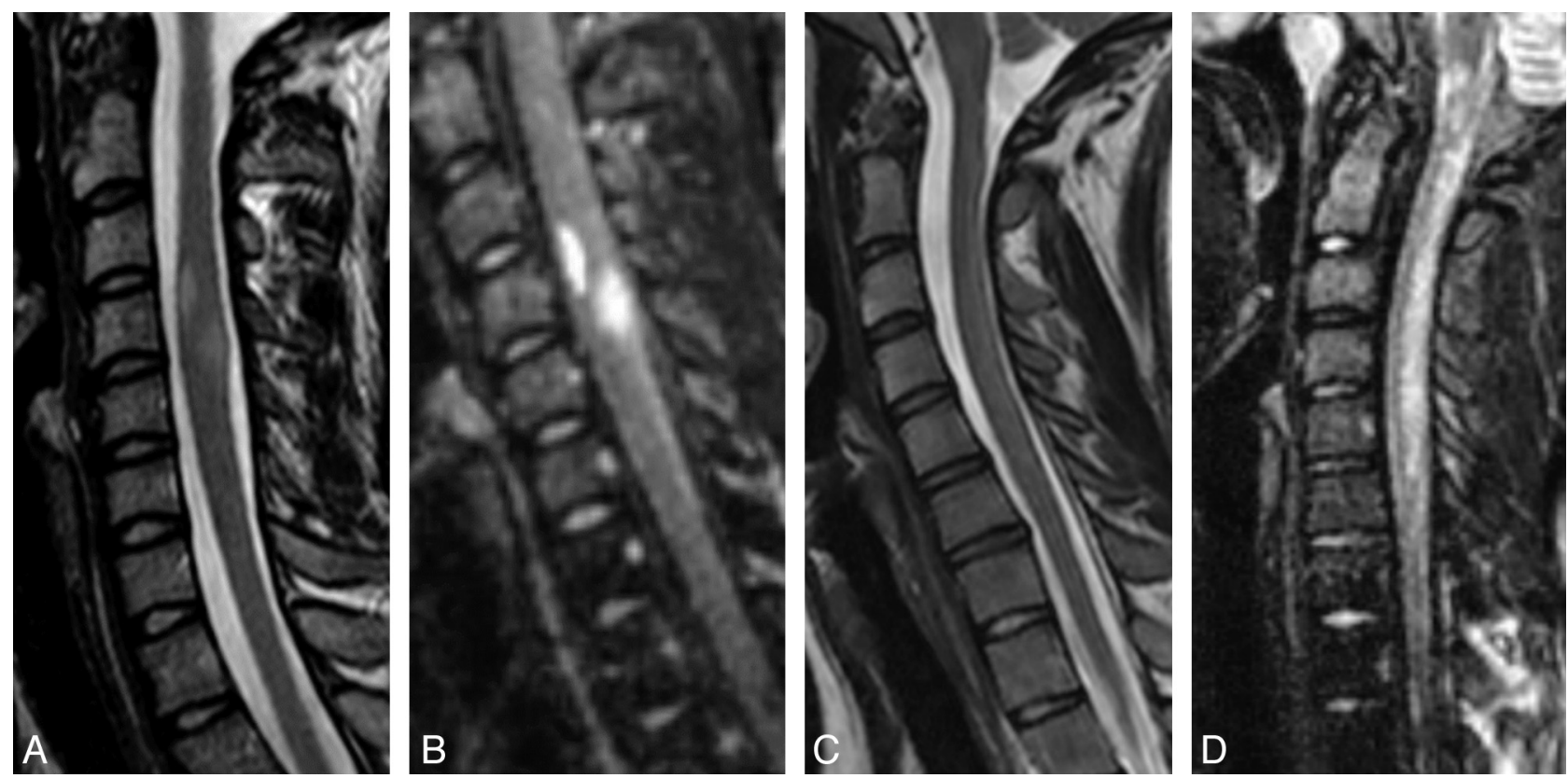

FIG 4. Sagittal T2WI TSE $(A$ and $C)$ and DIR $(B$ and $D)$ images of the cervical spinal cord of 2 patients. $A$ and $B$, Two lesions in the spinal cord at the $C 4$ vertebral body level of a 22-year-old female patient with clinically isolated syndrome. $C$ and $D$, A diffuse myelitis of an 18 -year-old female patient with MS. The lesion-to-background contrast sCNR in DIR images is remarkably higher.

More recently, alternative techniques, such as classic T2WI STIR, a modified TIWI STIR, and a 3D gradient recalled-echo sequence, have been applied to spinal cord imaging in MS. Although these sequences yielded promising results, comparing favorably with T2WI TSE sequences, ${ }^{5-8}$ current guidelines still include the conventional proton-attenuation/T2WI TSE sequence as part of the spinal cord imaging protocol in MS, ${ }^{4}$ which we therefore chose as a reference. However, future studies need to directly compare these sequences, all of which appear to be candidate techniques to replace or supplement conventional T2WI TSE sequences in future standard MS imaging protocols.

At present, the diagnosis of MS is usually based on the McDonald criteria, as revised in $2010,{ }^{22}$ which conceptually rely on the demonstration of lesion dissemination in both space and time. Both dissemination in space and dissemination in time can be evidenced by clinical findings and history, but also by MR imaging, ${ }^{22}$ following the simplified MR imaging criteria developed by Swanton et al. ${ }^{23}$ Herein, the detection of at least 1 lesion on spinal cord MR imaging in addition to at least 1 cerebral lesion will fulfill the criterion of dissemination in space, and the detection of any novel spinal lesion in follow-up MR imaging will fulfill the criterion of dissemination in time.

Hence, current diagnostic criteria for MS rely heavily on the detection of spatially segregated or novel lesions. Therefore, we focused on lesion counts to compare the sensitivities of 3D DIR and T2WI TSE imaging. Notwithstanding the authoritative role of the McDonald criteria in MS however, lesion numbers alone have limited informative value. Other parameters such as the lesion location, the overall volume of lesions, and the clinical impact are important and will influence patient management. Moreover, novel MR imaging-based techniques, including diffusion tensor, magnetization transfer, and "tissue-specific" imaging, may provide estimates of the degree of tissue damage in lesions; reveal abnormalities in healthy-appearing tissue, as shown in neuromyelitis optica; and help differentiate lesions with distinct pathologic substrates (demyelination, inflammation, edema, gliosis, remyelination) but similar intrinsic signal in T2WI sequences. ${ }^{9,10,24,25}$ Such techniques may provide valuable information beyond mere lesion visualization, and this may well be incorporated into future diagnostic guidelines.

Current diagnostic criteria, however, warrant the assessment of lesion-detection rates to compare imaging techniques in MS. Their clinical relevance is highlighted by 2 of the 18 (11\%) patients in our study, in whom a definite diagnosis had not been established before imaging. In 1 patient with a prominent lesion in the cervical cord, detection of a second lesion (conspicuous only in the 3D DIR image) largely ruled out a neoplastic process and favored the diagnosis of an inflammatory disease. In the other patient, several spinal lesions, revealed only by the 3D DIR sequence, completed the criterion of dissemination in space, changing the diagnosis from "possible" to "definite" MS.

In addition, the benefits of the 3D DIR sequence are likely not to be confined to improved lesion-detection rates. The high sCNR, in combination with high isotropic spatial resolution and the possibility of generating multiplanar reconstructions from a 3D dataset (eg, coronal planes, which are not commonly acquired in routine spinal MR imaging of patients with MS), may allow a more precise delineation of lesion borders, in particular of complex, elongated lesions and small lesions adjacent to the CSF (Fig 5). As one consequence, we would expect the 3D DIR sequence to provide a more precise tool for volumetric measurements than conventional 2D T2WI TSE sequences, which are prone to inaccuracies due to partial volume effects and lower contrast. This expectation, however, needs to be confirmed by additional studies.

We acknowledge limitations of our study. Most important, the

AJNR Am J Neuroradiol 36:219-25 Jan 2015 www.ajnr.org 

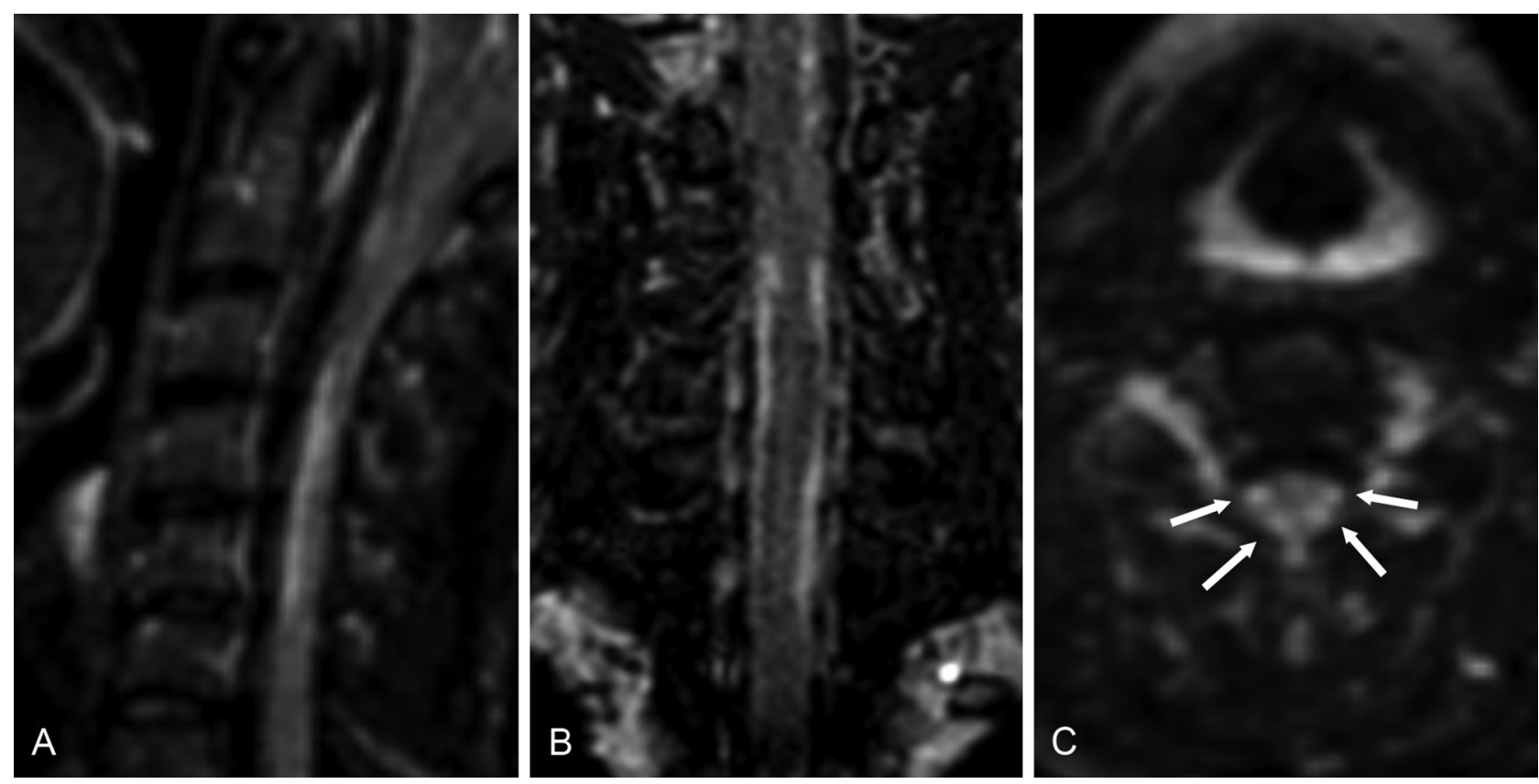

FIG 5. Sagittal $(A)$, coronal $(B)$, and axial (C) reconstructions of 3D DIR images of the cervical spinal cord of a 42 -year-old female patient with primary-progressive MS. Elongated lesions in the lateral spinal cord are visible on both sides (arrow).

spinal 3D DIR sequence was more strongly affected by artifacts and magnetic field inhomogeneities than conventional 2D T2WI TSE sequences. Overall, these were outweighed by still higher sCNR and consequently improved lesion-detection rates but led to unacceptable image quality in 3 patients with marked obesity. Moreover, the 3D DIR sequence of the thoracic spinal cord is technically even more challenging because of large magnetic susceptibility differences of adjacent tissues (lungs versus abdominal organs) and motion artifacts caused by cardiac pulsation and respiration. Preliminary studies of the thoracic spinal cord with a modified 3D DIR sequence yielded results comparable with those in the cervical spinal cord in selected patients (Fig 6). Nevertheless, at present, routine clinical application of the spinal 3D DIR sequence is confined to the cervical spinal cord, and further optimization is required to improve its stability to make it suitable for the lower part of the spinal cord as well. Furthermore, follow-up examinations were not available to determine whether lesions conspicuous only with the 3D DIR sequence would also become visible in conventional T2WI TSE images after some time. Finally, hyperintensities visible in the $3 \mathrm{D}$ DIR sequence are not specific for MS lesions, but this feature also holds true for conventional T2WI TSE sequences. One study correlating a postmortem 3D DIR sequence and histopathology showed the high specificity of the 3D DIR sequence for lesions in the cerebral cortex of patients with $\mathrm{MS}^{26}$ A similar MR imaging-to-histopathology correlation would be desirable for the spinal 3D DIR sequence but was not possible in the present study. However, no abnormalities were detected in the healthy volunteers in blinded analysis, so the risk of false-positive findings in the spinal 3D DIR sequence is likely to be very low.

\section{CONCLUSIONS}

The 3D DIR sequence allowed better detection of lesions in MS and related inflammatory diseases of the cervical spinal cord in
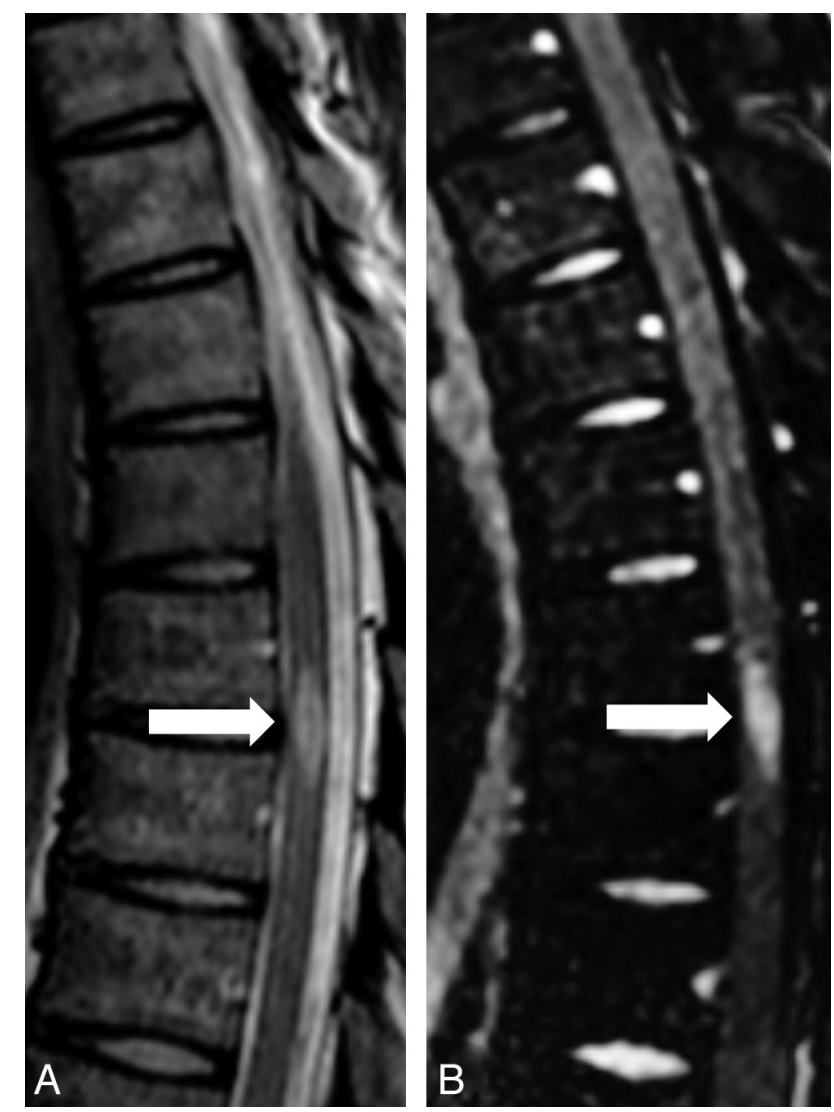

FIG 6. Sagittal T2WI TSE $(A)$ and DIR $(B)$ sequence of the thoracic spinal cord of a 35-year-old female patient with clinically isolated syndrome. On both sequences, a hyperintense lesion is visible in the spinal cord at the $\mathrm{T7}$ level (arrow).

comparison with conventional 2D T2WI TSE sequences. Currently, we recommend acquiring the 3D DIR sequence of the cervical spinal cord, especially in doubtful cases. 


\section{ACKNOWLEDGMENTS}

We thank Hendrik Kooijman (Philips Healthcare, Hamburg, Germany) for his support in establishing the 3D DIR sequence. Furthermore, we are grateful to all patients and volunteers who participated in this study.

Disclosures: Dimitrios C. Karampinos—UNRELATED: Grants/Grants Pending: Philips Healthcare.* Jan S. Bauer-UNRELATED: Grants/Grants Pending: German Research Foundation (DFG BA 4085 1/2)*; Travel/Accommodations/Meeting Expenses Unrelated to Activities Listed: meeting expenses covered by Terumo/MicroVention, United States. * Mark Mühlau—UNRELATED: Payment for Lectures (including service on Speakers Bureaus): Merck Serono*; Travel/Accommodations/Meeting Expenses Unrelated to Activities Listed: Bayer, Merck Serono. *Money paid to the institution.

\section{REFERENCES}

1. Bot JC, Barkhof F, Lycklama A, et al. Differentiation of multiple sclerosis from other inflammatory disorders and cerebrovascular disease: value of spinal MR imaging. Radiology 2002;223:46-56

2. Lukas C, Sombekke MH, Bellenberg B, et al. Relevance of spinal cord abnormalities to clinical disability in multiple sclerosis: MR imaging findings in a large cohort of patients. Radiology 2013;269:542-52

3. Sombekke MH, Wattjes MP, Balk LJ, et al. Spinal cord lesions in patients with clinically isolated syndrome: a powerful tool in diagnosis and prognosis. Neurology 2013;80:69-75

4. Simon JH, Li D, Traboulsee A, et al. Standardized MR imaging protocol for multiple sclerosis: consortium of MS Centers Consensus Guidelines. AJNR Am J Neuroradiol 2006;27:455-61

5. Bot JC, Barkhof F, Lycklama A, et al. Comparison of a conventional cardiac-triggered dual spin-echo and a fast STIR sequence in detection of spinal cord lesions in multiple sclerosis. Eur Radiol 2000; 10:753-58

6. Hittmair K, Mallek R, Prayer D, et al. Spinal cord lesions in patients with multiple sclerosis: comparison of MR pulse sequences. AJNR Am J Neuroradiol 1996;17:1555-65

7. Thorpe JW, MacManus DG, Kendall BE, et al. Short tau inversion recovery fast spin-echo (fast STIR) imaging of the spinal cord in multiple sclerosis. Magn Reson Imaging 1994;12:983-89

8. Ozturk A, Aygun N, Smith SA, et al. Axial 3D gradient-echo imaging for improved multiple sclerosis lesion detection in the cervical spinal cord at 3T. Neuroradiology 2013;55:431-39

9. Rivero RL, Oliveira EM, Bichuetti DB, et al. Diffusion tensor imaging of the cervical spinal cord of patients with neuromyelitis optica. Magn Reson Imaging 2014;32:457-63

10. Naismith RT, Xu J, Klawiter EC, et al. Spinal cord tract diffusion tensor imaging reveals disability substrate in demyelinating disease. Neurology 2013;80:2201-09

11. Redpath TW, Smith FW. Imaging gray brain matter with a double- inversion pulse sequence to suppress CSF and white matter signals. MAGMA 1994;2:451-55

12. Redpath TW, Smith FW. Technical note: use of a double inversion recovery pulse sequence to image selectively grey or white brain matter. Br J Radiol 1994;67:1258-63

13. Bedell BJ, Narayana PA. Implementation and evaluation of a new pulse sequence for rapid acquisition of double inversion recovery images for simultaneous suppression of white matter and CSF. $J$ Magn Reson Imaging 1998;8:544-47

14. Calabrese M, De Stefano N, Atzori M, et al. Detection of cortical inflammatory lesions by double inversion recovery magnetic resonance imaging in patients with multiple sclerosis. Arch Neurol 2007;64:1416-22

15. Geurts JJ, Pouwels PJ, Uitdehaag BM, et al. Intracortical lesions in multiple sclerosis: improved detection with 3D double inversionrecovery MR imaging. Radiology 2005;236:254-60

16. Wattjes MP, Lutterbey GG, Gieseke J, et al. Double inversion recovery brain imaging at $3 \mathrm{~T}$ : diagnostic value in the detection of multiple sclerosis lesions. AJNR Am J Neuroradiol 2007;28:54-59

17. Shipp D. Case report: cervical spine $3 \mathrm{D}$ double inversion recovery (DIR) in demyelination. MAGNETOM Flash 2012:49-50. www. siemens.com/magnetom-world. Accessed October 10, 2013

18. Edelstein WA, Glover GH, Hardy CJ, et al. The intrinsic signal-tonoise ratio in NMR imaging. Magn Reson Med 1986;3:604-18

19. Lin LI. A concordance correlation coefficient to evaluate reproducibility. Biometrics 1989;45:255-68

20. Keiper MD, Grossman RI, Brunson JC, et al. The low sensitivity of fluid-attenuated inversion-recovery MR in the detection of multiple sclerosis of the spinal cord. AJNR Am J Neuroradiol 1997;18: 1035-39

21. Thomas DJ, Pennock JM, Hajnal JV, et al. Magnetic resonance imaging of spinal cord in multiple sclerosis by fluid-attenuated inversion recovery. Lancet 1993;341:593-94

22. Polman $\mathrm{CH}$, Reingold SC, Banwell B, et al. Diagnostic criteria for multiple sclerosis: 2010 revisions to the McDonald criteria. Ann Neurol 2011;69:292-302

23. Swanton JK, Rovira A, Tintore M, et al. MRI criteria for multiple sclerosis in patients presenting with clinically isolated syndromes: a multicentre retrospective study. Lancet Neurol 2007;6:677-86

24. Bagnato F, Ikonomidou VN, van Gelderen P, et al. Lesions by tissue specific imaging characterize multiple sclerosis patients with more advanced disease. Mult Scler 2011;17:1424-31

25. Rovira A. Tissue-specific MR imaging in multiple sclerosis. AJNR Am J Neuroradiol 2009;30:1277-78

26. Seewann A, Kooi EJ, Roosendaal SD, et al. Postmortem verification of MS cortical lesion detection with 3D DIR. Neurology 2012;78: 302-08 